\title{
KRAS-mutated non-small cell lung cancer cells are responsive to either co-treatment with erlotinib or gefitinib and histone deacetylase inhibitors or single treatment with lapatinib
}

\author{
INA KURTZE, JÜRGEN SONNEMANN and JAMES F. BECK \\ University Children's Hospital Jena, Department of Pediatric Hematology and Oncology, Jena, Germany
}

Received October 19, 2010; Accepted December 16, 2010

DOI: $10.3892 /$ or.2011.1160

\begin{abstract}
The epidermal growth factor receptor (EGFR) tyrosine kinase inhibitors erlotinib and gefitinib provide significant clinical benefit for non-small cell lung cancer (NSCLC) patients whose tumors bear EGFR mutations/ amplifications. However, anti-EGFR therapy is largely ineffective in NSCLC with activating KRAS mutations. In this study, we investigated the treatment efficacy of erlotinib and gefitinib in combination with the histone deacetylase inhibitors (HDACi) vorinostat and sodium butyrate in the KRAS-mutated NSCLC cell line A549. For comparison, we tested the combination of HDACi with the dual tyrosine kinase inhibitor lapatinib. A549 cells proved to be resistant to erlotinib and gefitinib, but could be sensitized by cotreatment with HDACi, as assessed by flow cytometric analyses of cell death and mitochondrial depolarization. In contrast, A549 cells were a priori responsive to lapatinib treatment, but responsiveness to lapatinib could not be enhanced by HDACi cotreatment. These divergent effects of the different combination regimens may be explained by dissimilar types of cell death induced by the treatments: The use of the pan-caspase inhibitor z-VAD-fmk in the cell death and mitochondrial depolarization assays as well as fluorescence microscopy analyses indicated that erlotinib or gefitinib combined with HDACi elicited apoptosis, whereas lapatinib treatment induced a non-apoptotic type of cell death. Our study suggests that both HDACi/EGFR inhibitor-combination treatment and lapatinib-single treatment may be effective options for the therapy of NSCLC with KRAS mutations.
\end{abstract}

\section{Introduction}

Lung cancer is the leading cause of death from cancer worldwide. Non-small cell lung cancer (NSCLC) accounts for

Correspondence to: Dr Jürgen Sonnemann, Klinik für Kinderund Jugendmedizin, Friedrich-Schiller-Universität Jena, Kochstr. 2, D-07745 Jena, Germany

E-mail: juergen.sonnemann@med.uni-jena.de

Key words: apoptosis, erlotinib, gefitinib, histone deacetylase inhibitors, lapatinib, non-apoptotic cell death, non-small cell lung cancer
$85 \%$ of all lung cancers; in 40-80\% of NSCLC cases, overexpression of the epidermal growth factor receptor (EGFR) is observed (1). EGFR is a member of the HER family of receptor tyrosine kinases; other EGFR family members are HER2, HER3 and HER4 (2). Activated EGFR triggers the RAS-MAPK pathway, which controls gene transcription and cell proliferation, and the PI3K-Akt pathway, which induces prosurvival signals. Activation of these pathways may result in tumor progression.

Tyrosine kinase inhibitors (TKI), such as erlotinib, gefitinib and lapatinib, inhibit EGFR activation and downstream signaling. In preclinical cancer models, blocking of EGFR by TKI prevents the proliferation of cancer cells (1). Clinical studies have demonstrated favorable response to EGFR TKI particularly in patients with lung cancer harboring EGFR mutations (3). Consequently, erlotinib and gefitinib have been approved by the US Food and Drug Administration (FDA) for the treatment of chemotherapy-resistant NSCLC. However, $15-30 \%$ of patients with NSCLC bear activating mutations in the KRAS gene (2). Because RAS is downstream from EGFR, activating KRAS mutations frequently confer resistance to EGFR TKI. In fact, clinical studies have shown that tumors with KRAS mutations rarely respond to EGFR TKI therapy (4). A dual kinase inhibitor of EGFR and HER2 tyrosine kinases, lapatinib (5), has been approved by the FDA for the treatment of advanced breast cancer overexpressing HER2.

Histone deacetylase inhibitors (HDACi), such as vorinostat (also known as suberoylanilide hydroxamic acid, SAHA), are another class of antineoplastic agents that hold promise to improve cancer therapy $(6,7)$. They stimulate differentiation, inhibit proliferation and induce apoptosis in tumor cells while leaving normal cells rather unaffected. Their clinical potential is highlighted by the fact that vorinostat, and more recently romidepsin, have been approved by the FDA for treatment of cutaneous T-cell lymphoma. However, the greatest potential of HDACi may lie in their capability to enhance the anticancer efficacy of other therapeutic regimens. In numerous preclinical studies, they have been demonstrated to synergize with a multitude of pharmacological and biological cancer therapeutics (8). For instance, we have previously shown that HDACi interact favorably with agents as diverse as aspirin (9), the apoptosis-inducing cytokine TRAIL (10) and the p53 activator nutlin-3 (11) to elicit cancer cell death. With 

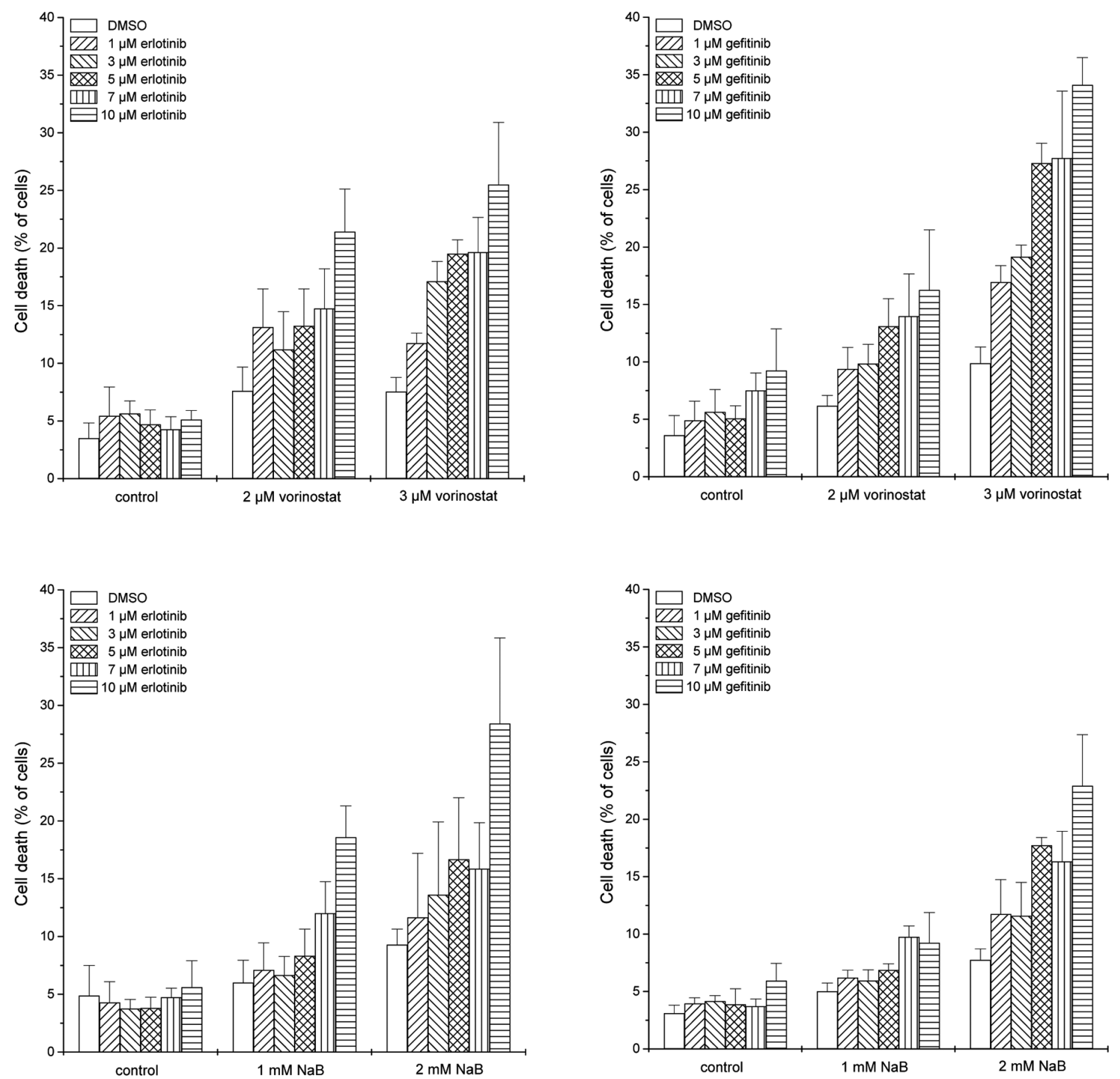

Figure 1. HDACi and EGFR TKI cooperate in inducing cell death in A549 cells. Four hours after administration of HDACi, cells were exposed to erlotinib or gefitinib for another $48 \mathrm{~h}$. Cell death was determined by flow cytometric analysis of PI uptake. Means \pm SEM of each 3 (vorinostat) or 4 (NaB) separate experiments are shown.

respect to the combination of EGFR TKI with HDACi in NSCLC cells, erlotinib has been reported to cooperate with panobinostat and romidepsin $(12,13)$, and gefitinib has been reported to cooperate with vorinostat and MS-275 $(14,15)$; the combination of HDACi with lapatinib has not yet been tested.

In this study, we investigated whether HDACi belonging to two different structural classes, the hydroxamic acid vorinostat and the short chain fatty acid sodium butyrate $(\mathrm{NaB})$, could sensitize KRAS-mutated A549 NSCLC adenocarcinoma cells to erlotinib and/or gefitinib. Since A549 cells express HER2 $(16,17)$, we also tested the combination of HDACi with lapatinib. We found A549 cells to be resistant to erlotinib and gefitinib, but responsive to lapatinib. Erlotinib and gefitinib resistance could be overcome by cotreatment with HDACi.

\section{Materials and methods}

Reagents. Erlotinib, gefitinib and lapatinib were purchased from LC Laboratories (Woburn, MA, USA). Vorinostat and the pan-caspase inhibitor $\mathrm{z}$-VAD-fmk were purchased from Alexis (Grünberg, Germany). NaB was purchased from Sigma-Aldrich (Deisenhofen, Germany).

Cell lines. A549 cells were obtained from ATCC (Manassas, VA, USA). Cells were maintained in Ham's F12K medium supplemented with $10 \%$ fetal calf serum, $2 \mathrm{mM} \mathrm{L-glutamine,}$ $100 \mathrm{U} / \mathrm{ml}$ penicillin $\mathrm{G}$ sodium and $100 \mu \mathrm{g} / \mathrm{ml}$ streptomycin sulfate (PAA, Cölbe, Germany). Cells were cultivated at $37^{\circ} \mathrm{C}$ in a humidified $5 \% \mathrm{CO}_{2}$ incubator and routinely passaged when $90 \%$ confluent. Cell viability was determined by the 
Table I. Combination index values for vorinostat plus erlotinib.

\begin{tabular}{lcc}
\hline Vorinostat $(\mu \mathrm{M})$ & Erlotinib $(\mu \mathrm{M})$ & $\mathrm{CI}$ \\
\hline 2 & 1 & 0.618 \\
2 & 3 & 0.548 \\
2 & 5 & 0.520 \\
2 & 7 & 0.437 \\
2 & 10 & 0.399 \\
3 & 1 & 0.864 \\
3 & 3 & 0.662 \\
3 & 5 & 0.600 \\
3 & 7 & 0.597 \\
3 & 10 & 0.487 \\
\hline
\end{tabular}

Based on data from Fig. 1, CI values were calculated using the ChouTalalay method.

trypan blue exclusion test. Cells were regularly inspected to be free of mycoplasma with the PCR mycoplasma detection kit from Applichem (Darmstadt, Germany).

Treatment of cells. The cells were plated at 100,000 cells/ well in 6-well plates and treated with HDACi for $4 \mathrm{~h}$ or left untreated before application of TKI. The latter were added directly to the culture medium containing HDACi without a medium change. Cells were then exposed to TKI for additional 24 (fluorescence microscopy) or $48 \mathrm{~h}$ (flow cytometric analyses). To inhibit the activation of caspases, z-VAD-fmk was applied $1 \mathrm{~h}$ before administration of HDACi.

Flow cytometric analysis of cell death. Cell death was assessed by determining the integrity of the cell membrane by flow cytometric analysis of propidium iodide (PI) uptake. After harvesting, cells were incubated for $5 \mathrm{~min}$ in $2 \mu \mathrm{g} / \mathrm{ml}$ PI in PBS at $4^{\circ} \mathrm{C}$ in the dark and PI uptake was measured on a BD (Heidelberg, Germany) FACSCanto II. Ten thousand cells were analyzed in each sample; data were gated to exclude debris. The results from the assays were analyzed by the combination index (CI) method according to Chou and Talalay (18) using Calcusyn software from Biosoft (Cambridge, UK). CI values of $<1,=1$ and $>1$ indicate synergism, additivism and antagonism, respectively.

Flow cytometric analysis of mitochondrial transmembrane potential $\left(\Delta \psi_{m}\right) . \Delta \psi_{\mathrm{m}}$ was determined by assessing the accumulation of 3,3'-dihexyloxacarbocyanine iodide [ $\mathrm{DiOC}_{6}$ (3)] in the mitochondrial matrix. Before harvesting, cells were incubated with $50 \mathrm{nM} \mathrm{DiOC}_{6}$ (3) (Molecular Probes, Eugene, OR, USA) at $37^{\circ} \mathrm{C}$ for $30 \mathrm{~min}$. After washing, 10,000 cells were analyzed using the FACSCanto II. Data were gated to exclude debris.

Fluorescence microscopy. Cells were plated at 50,000 cells/ well on coverslips in 6-well plates. At the end of the treatment period, cells were either double stained with $1 \mu \mathrm{g} / \mathrm{ml}$ PI and $2 \mu \mathrm{g} / \mathrm{ml}$ Hoechst 34580 (Invitrogen, Darmstadt, Germany)
Table II. Combination index values for vorinostat plus gefitinib.

\begin{tabular}{lcc}
\hline Vorinostat $(\mu \mathrm{M})$ & Gefitinib $(\mu \mathrm{M})$ & $\mathrm{CI}$ \\
\hline 2 & 1 & 0.765 \\
2 & 3 & 0.807 \\
2 & 5 & 0.609 \\
2 & 7 & 0.585 \\
2 & 10 & 0.508 \\
3 & 1 & 0.679 \\
3 & 3 & 0.615 \\
3 & 5 & 0.445 \\
3 & 7 & 0.439 \\
3 & 10 & 0.357 \\
\hline
\end{tabular}

Based on data from Fig. 1, CI values were calculated using the ChouTalalay method.

or single stained with $50 \mathrm{nM} \mathrm{DiOC}{ }_{6}(3)$. Fluorescences were detected using excitation/emission wave lengths of 561/580-750 nm (PI), 405/410-500 nm (Hoechst 34580) and 488/500-600 nm [DiOC 6 (3)]. Cells were viewed using a Leica (Wetzlar, Germany) TCS SP5 confocal laser scanning microscope, and image analysis was done by using Leica Application Suite imaging software.

\section{Results}

HDACi sensitizes KRAS-mutated A549 NSCLC cells to erlotinib and gefitinib. To assess a possible favorable interaction between EGFR TKI and HDACi, we initially monitored cell death in A549 cells by flow cytometric analysis of PI uptake. A549 cells harbor a KRAS mutation (19) and have a very low sensitivity to EGFR TKI $(20,21)$. Fig. 1 shows that the cells were indeed resistant to erlotinib and gefitinib at the concentrations applied. Likewise, vorinostat and $\mathrm{NaB}$-affected cell viability only weakly. However, when erlotinib or gefitinib were applied in conjunction with HDACi, they evoked cell death in a concentration-dependent manner. For example, when administered individually, $3 \mu \mathrm{M}$ vorinostat and $10 \mu \mathrm{M}$ erlotinib elicited cell death in 8 or $5 \%$ of cells, respectively. When applied together, the agents evoked cell death in $26 \%$ of cells. To test for synergy, we analyzed these data by the CI method $(\mathrm{CI}<1$ is indicative for a synergistic interaction) (18). The calculated CI values indicated synergism for all combinations tested (Tables I-IV).

Cell death induced by the combination of HDACi with erlotinib or gefitinib involves apoptosis. To analyze whether the cooperative action of erlotinib and gefitinib with HDACi stemmed from the cooperative induction of apoptosis, we assessed the mitochondrial membrane potential $\left(\Delta \psi_{\mathrm{m}}\right)$ and applied the pan-caspase inhibitor z-VAD-fmk. Since the intrinsic apoptotic pathway involves a perturbation of $\Delta \psi_{\mathrm{m}}$, we first determined $\Delta \psi_{\mathrm{m}}$ dissipation by flow cytometric analysis of $\mathrm{DiOC}_{6}(3)$ staining. As presented in Fig. 2A, the results reflect those of the cell death assays: erlotinib and 
Table III. Combination index values for $\mathrm{NaB}$ plus erlotinib.

\begin{tabular}{lcc}
\hline $\mathrm{NaB}(\mathrm{mM})$ & Erlotinib $(\mu \mathrm{M})$ & $\mathrm{CI}$ \\
\hline 1 & 1 & 0.769 \\
1 & 3 & 0.861 \\
1 & 5 & 0.597 \\
1 & 7 & 0.327 \\
1 & 10 & 0.153 \\
2 & 1 & 0.688 \\
2 & 3 & 0.530 \\
2 & 5 & 0.372 \\
2 & 7 & 0.407 \\
2 & 10 & 0.136 \\
\hline
\end{tabular}

Based on data from Fig. 1, CI values were calculated using the ChouTalalay method.
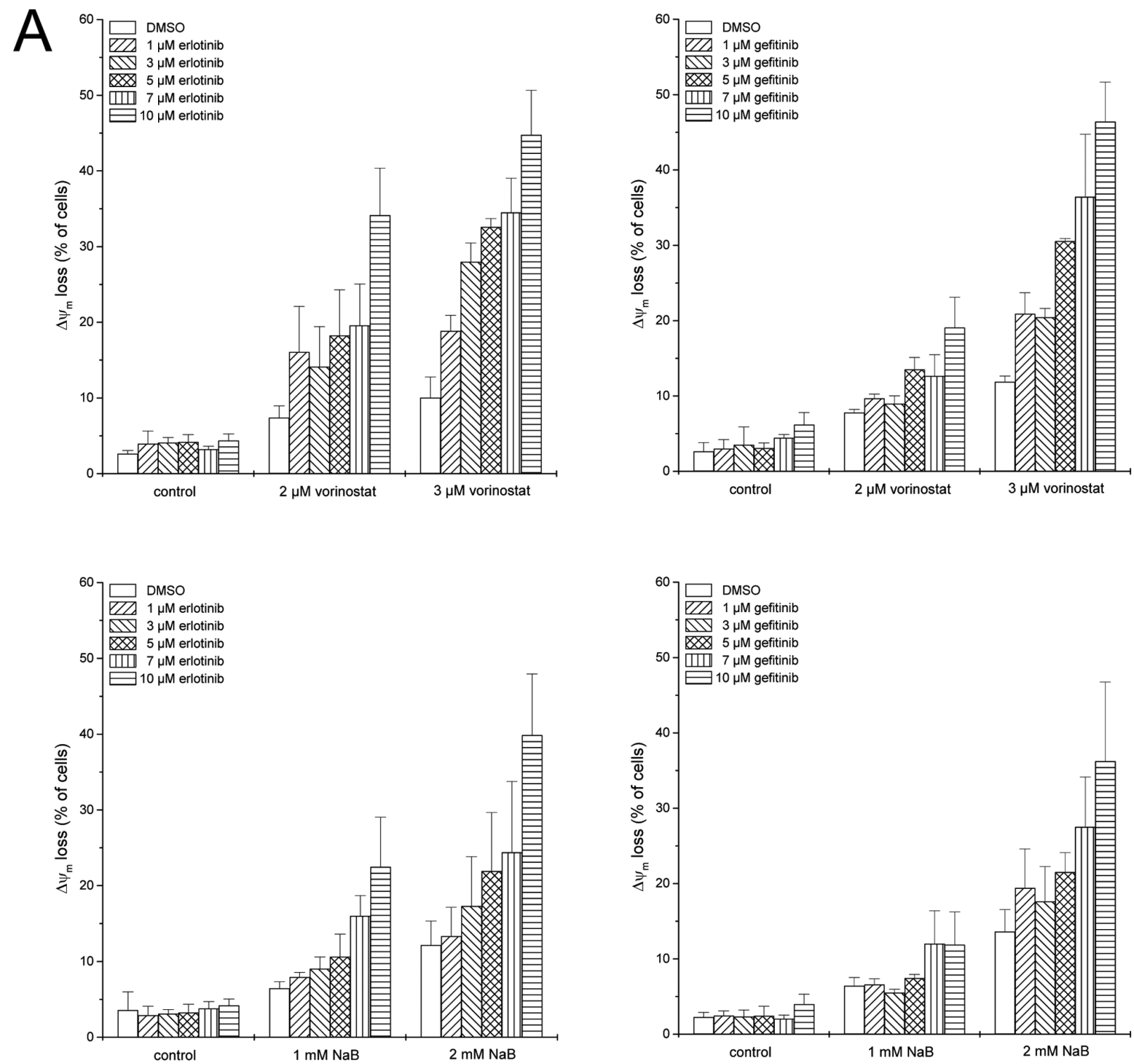

Table IV. Combination index values for $\mathrm{NaB}$ plus gefitinib.

\begin{tabular}{lcc}
\hline $\mathrm{NaB}(\mathrm{mM})$ & Gefitinib $(\mu \mathrm{M})$ & $\mathrm{CI}$ \\
\hline 1 & 1 & 0.719 \\
1 & 3 & 0.790 \\
1 & 5 & 0.616 \\
1 & 7 & 0.345 \\
1 & 10 & 0.377 \\
2 & 1 & 0.507 \\
2 & 3 & 0.518 \\
2 & 5 & 0.248 \\
2 & 7 & 0.288 \\
2 & 10 & 0.154 \\
\hline
\end{tabular}

Based on data from Fig. 1, CI values were calculated using the ChouTalalay method. 

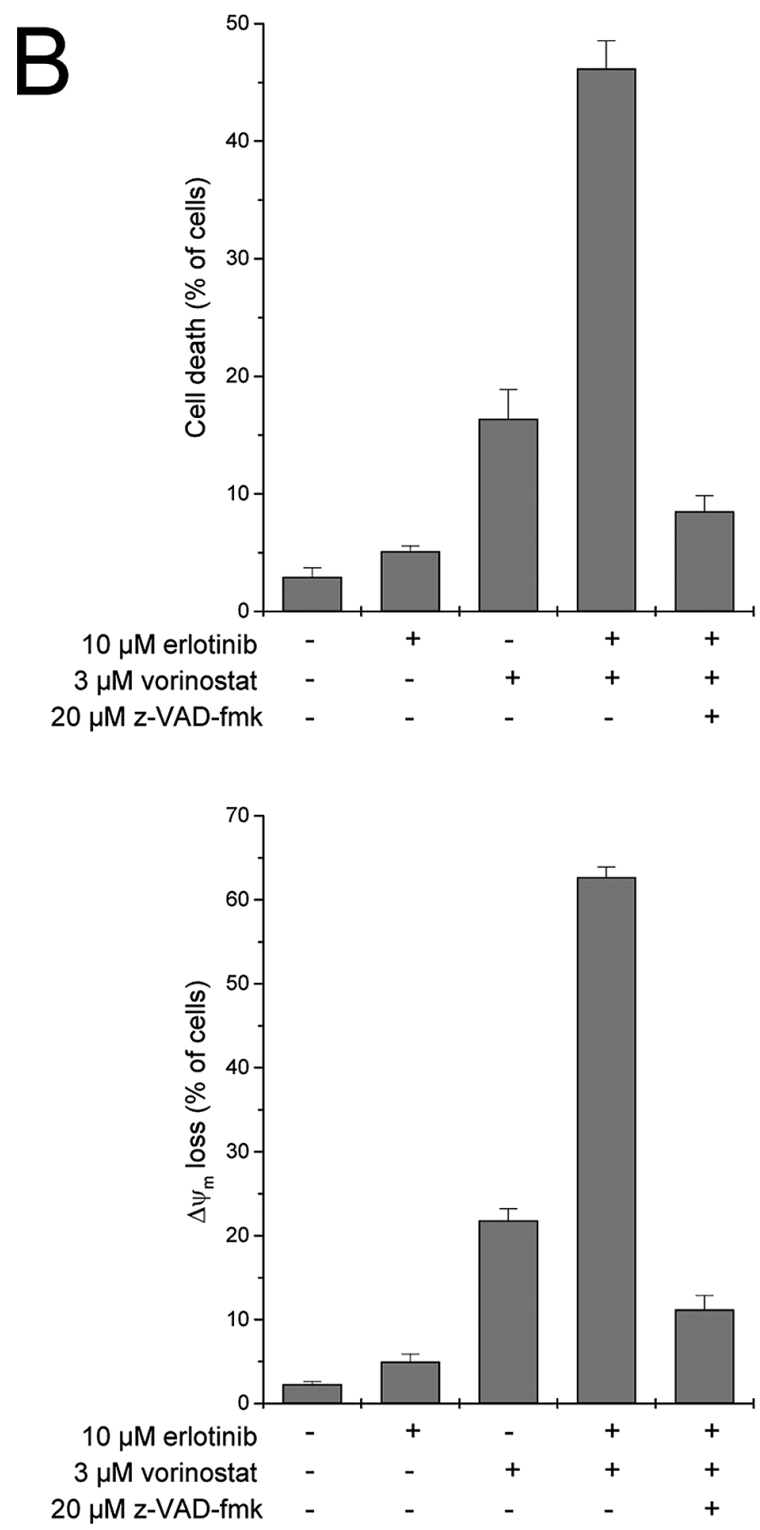
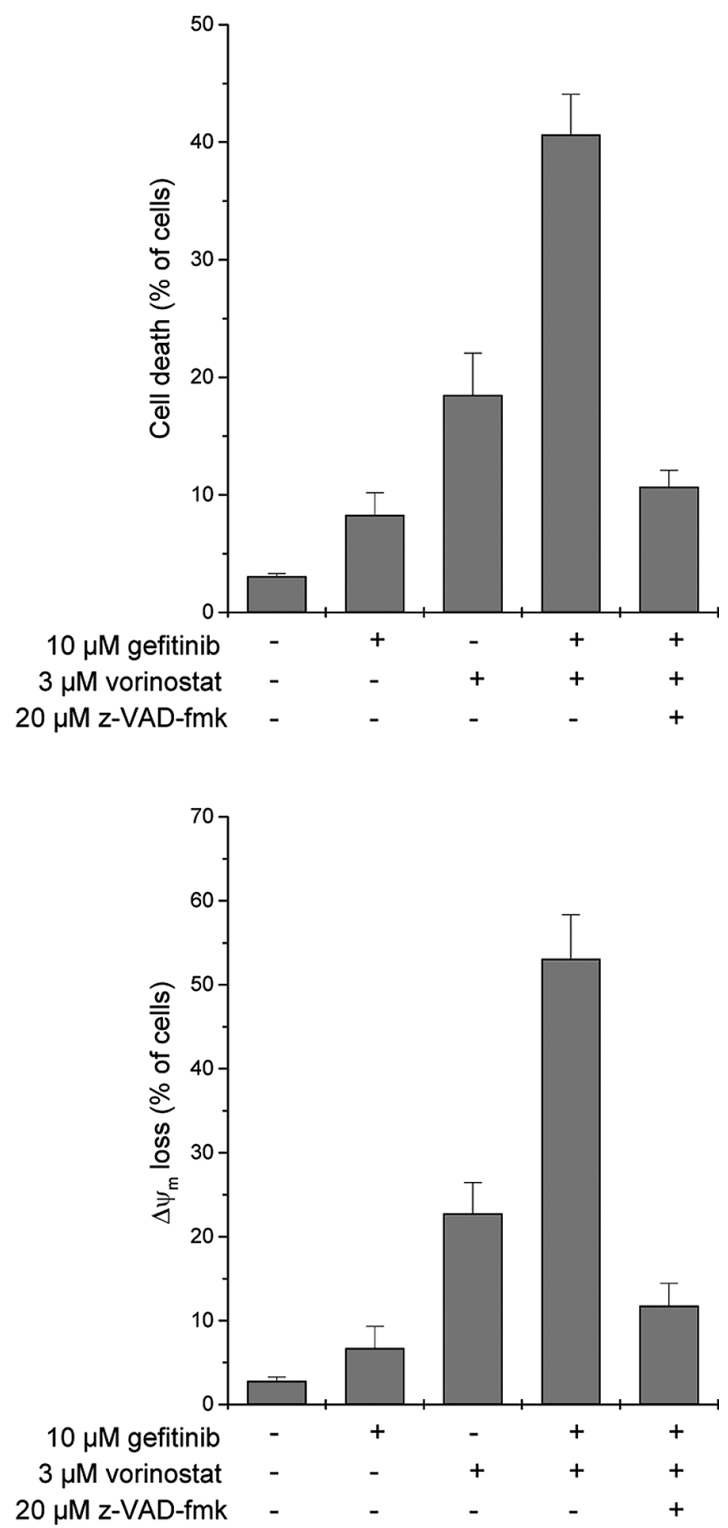

Figure 2. (B) z-VAD-fmk was applied $1 \mathrm{~h}$ before treatment with HDACi. Cell death and $\Delta \psi_{\mathrm{m}}$ dissipation were determined by flow cytometric analyses of PI uptake or $\mathrm{DiOC}_{6}(3)$ staining, respectively. Means \pm SEM of each 3 (vorinostat) or $4(\mathrm{NaB})$ separate experiments are shown.

gefitinib applied as single agents had no effect on $\Delta \psi_{\mathrm{m}}$ whereas in cells pre-exposed to HDACi, they induced a decay of $\Delta \psi_{\mathrm{m}}$ in a dose-dependent fashion. To explore whether the synergistic action of HDACi and TKI involved caspases, we applied z-VAD-fmk in the cell death and the $\Delta \psi_{\mathrm{m}}$ analyses. Fig. 2B shows that the inhibition of caspase activities strongly reduced the effects of the combination treatments both on cell death and $\Delta \psi_{\mathrm{m}}$ dissipation.

Lapatinib induces cell death, but not $\Delta \psi_{m}$ dissipation in A549 cells. For comparison, we tested A549 cells for their responsiveness to the dual kinase (EGFR and HER2) inhibitor lapatinib in combination with HDACi. Fig. 3A demonstrates that lapatinib alone triggered cell death in a dose-dependent manner, as determined by PI uptake analysis. However, the addition of HDACi to lapatinib did not produce a synergistic but rather additive effect. In stark contrast, lapatinib alone did not affect $\Delta \psi_{\mathrm{m}}$, while the combination treatment with HDACi and lapatinib resulted in $\Delta \psi_{\mathrm{m}}$ loss (Fig. 3B). Interestingly, z-VAD-fmk protected cells from HDACi/lapatinib-induced $\Delta \psi_{\mathrm{m}}$ dissipation, but not cell death (Fig. 3C).

Fluorescence microscopy indicates different types of cell death. These experiments suggested that lapatinib alone as well as the combination of lapatinib with HDACi induced a different mode of cell death from erlotinib or gefitinib in combination with HDACi. Therefore, to distinguish apoptotic cells from cells undergoing other types of cell death, we examined cells for morphological changes in the nuclear chromatin by double staining with the fluorescent dyes PI and Hoechst 34580. Intact blue nuclei, fragmented blue nuclei, fragmented pink nuclei and intact pink nuclei are indicative for viable, apoptotic, apoptotic/necrotic and necrotic cells, respectively (22). Fig. 4A shows that vorinostat/erlotinib combination treatment resulted in apoptotic morphology (nuclear fragmentation, white arrows), lapatinib treatment resulted in necrotic 

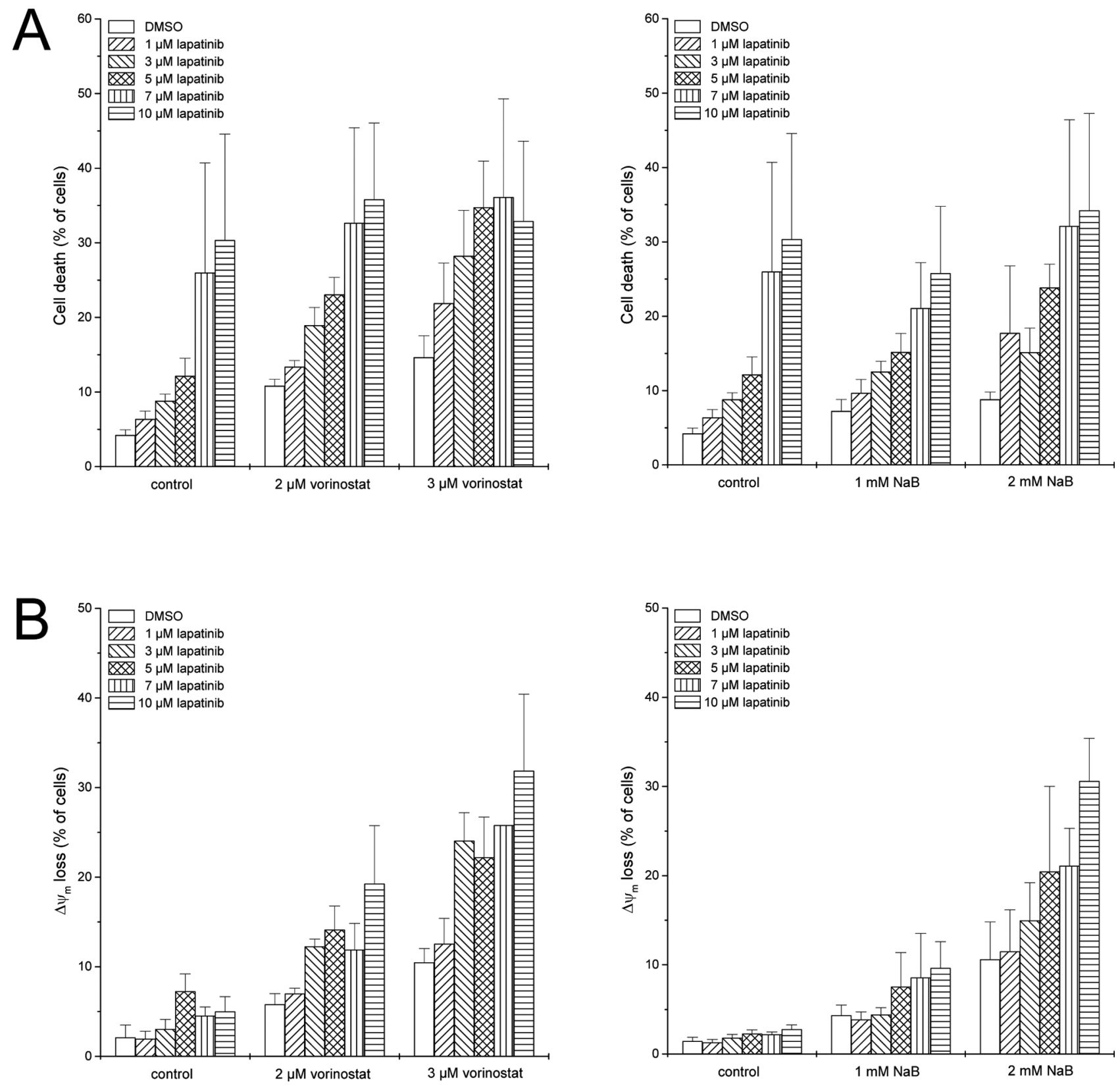

Figure 3. Lapatinib induces cell death in A549 cells. Four hours after administration of HDACi, cells were exposed to lapatinib for another 48 h. (A) Cell death was determined by flow cytometric analysis of PI uptake. (B) $\Delta \psi_{\mathrm{m}}$ was assessed by flow cytometric analysis of DiOC $\mathrm{D}_{6}(3)$ staining. $^{2}$.

morphology (loss of membrane integrity, red arrows), and vorinostat/lapatinib combination treatment resulted in apoptotic/necrotic morphology, indicated by nuclear fragmentation (red arrows) and loss of plasma membrane integrity (white arrows).

In addition, we examined the effects of TKI single treatment and HDACi/TKI combination treatment on mitochondrial morphology using $\operatorname{DiOC}_{6}(3)$ (23). The swelling of cell organelles, such as mitochondria, is an indication for nonapoptotic cell death (24). As illustrated in Fig. 4B, lapatinib and vorinostat/lapatinib treatment produced mitochondrial swelling. Of note, lapatinib displays visible red autofluorescence. In concordance with the flow cytometric analysis of DiOC $_{6}(3)$ staining, we observed a strong reduction of the fluorescence signal after vorinostat/lapatinib combination treatment. This is not perceptible, because fluorescence inten- sity was adjusted for optimum presentation of mitochondrial morphology.

\section{Discussion}

In this study, we have shown that the resistance of KRASmutated NSCLC cells to the EGFR TKI erlotinib and gefitinib could be overcome by combination treatment with HDACi. We have demonstrated that the TKI and HDACi interacted to induce cell death, and CI analysis evidenced that this interaction was synergistic. Erlotinib and gefitinib are in use for the treatment of patients with NSCLC harboring activating EGFR mutations (2). However, clinical trials have shown that NSCLC with KRAS mutations rarely respond to TKI therapy (4). Hence, in concordance with other in vitro studies (12-15), our findings suggest that the combination of EGFR TKI with 

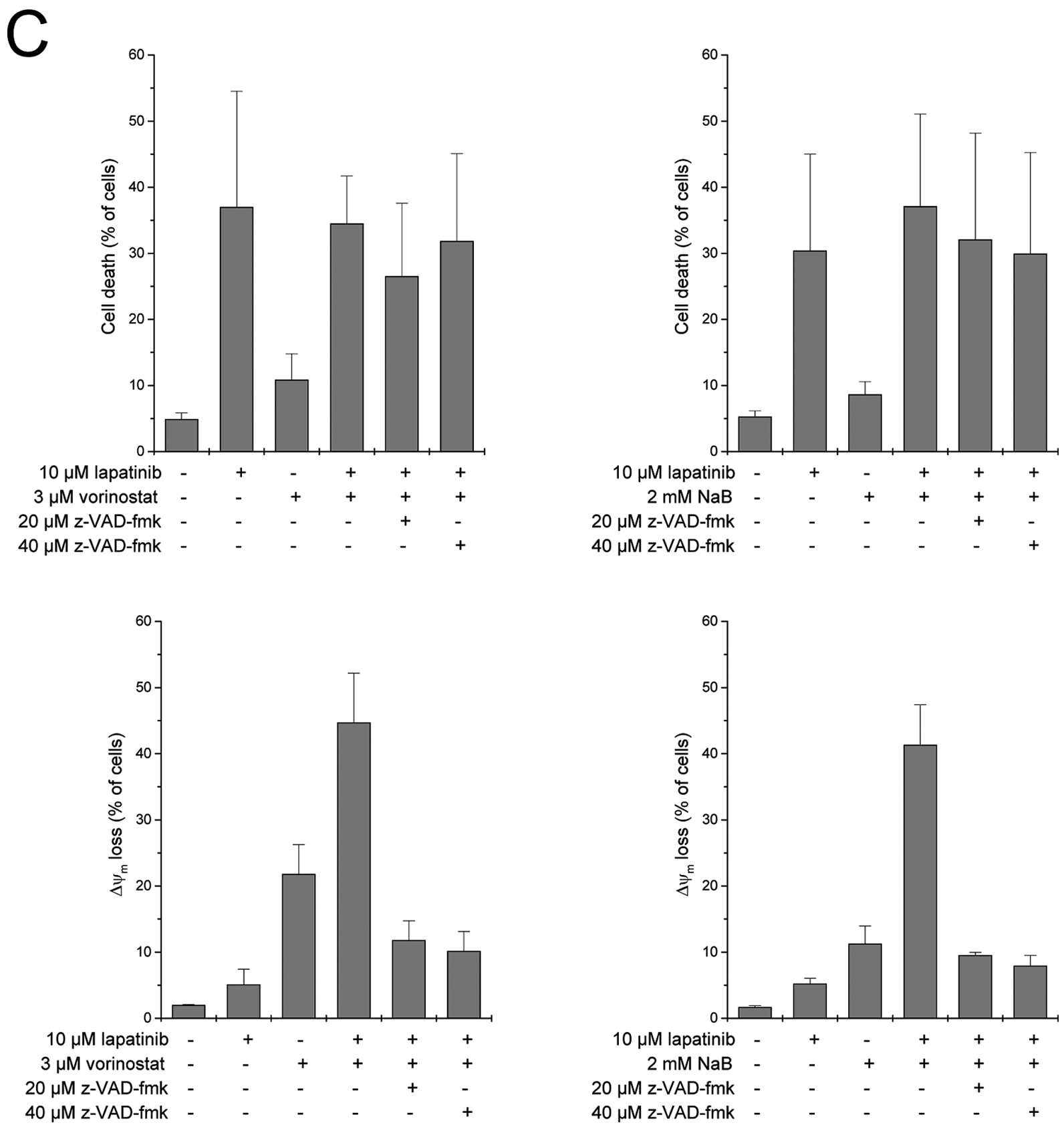

Figure. 3 (C) z-VAD-fmk was applied $1 \mathrm{~h}$ before treatment with HDACi. Cell death and $\Delta \psi_{\mathrm{m}}$ dissipation were determined by flow cytometric analyses of PI uptake or $\mathrm{DiOC}_{6}(3)$ staining, respectively. Means $\pm \mathrm{SEM}$ of each 4 (PI uptake) or $3\left[\mathrm{DiOC}_{6}(3)\right.$ staining] separate experiments are shown.

HDACi may provide a useful strategy for the treatment of KRAS-mutated NSCLC.

Like the majority of antineoplastic agents, EGFR TKI and HDACi elicit cell death through the induction of apoptosis $(25,26)$. Our results indicate that the combination of erlotinib or gefitinib with HDACi resulted in the collaborative initiation of apoptosis. First, we observed that the TKI in conjunction with HDACi cooperatively induced a decay of $\Delta \psi_{\mathrm{m}}$, a feature characteristic of apoptosis. In fact, after a given treatment period, the percentage of cells undergoing $\Delta \psi_{\mathrm{m}}$ dissipation systematically exceeded the percentage of cells undergoing cell death, indicating that $\Delta \psi_{\mathrm{m}}$ dissipation preceded cell death. Second, the use of the polycaspase inhibitor z-VADfmk revealed that caspase activity was required for HDACi/
TKI-induced cell death. The inhibition of caspases abolished the effects of the combination therapies on both cell death and loss of $\Delta \psi_{\mathrm{m}}$.

For comparison to the EGFR selective TKI erlotinib and gefitinib, we tested A549 cells for their responsiveness to the dual kinase inhibitor lapatinib (5). In concordance with recents reports $(16,17)$, we found A549 cells to be sensitive to lapatinib, suggesting that it may be an option for the treatment of erlotinib- or gefitinib-resistant NSCLC. However, in contrast to the combination of HDACi with erlotinib or gefitinib, the combination of HDACi with lapatinib did not produce a synergistic but rather additive effect. This finding implies that HDACi do not unselectively synergize with TKI irrespective of their mode of action. 

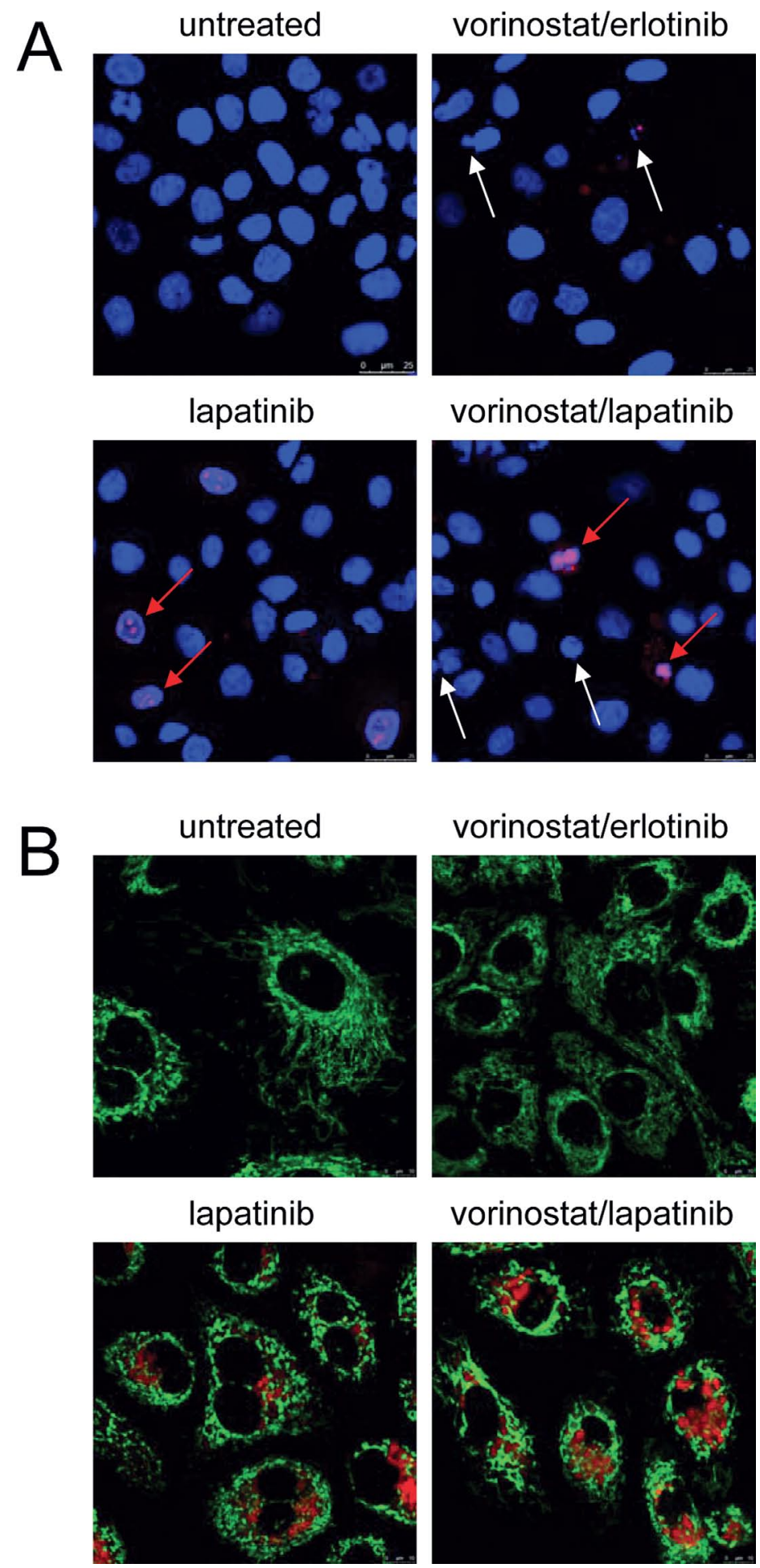

Figure 4. Lapatinib induces non-apoptotic cell death in A549 cells. Four hours after administration of $3 \mu \mathrm{M}$ vorinostat, cells were exposed to $10 \mu \mathrm{M}$ erlotinib or lapatinib for another $24 \mathrm{~h}$. (A) Cells were double-stained with PI/Hoechst 34580 and representative images were captured by fluorescence microscopy (scale bars, $25 \mu \mathrm{m}$ ). (B) Cells were stained with $\mathrm{DiOC}_{6}(3)$ and representative images were captured by fluorescence microscopy (scale bars, $10 \mu \mathrm{m}$ ).

Indeed, our data point to a non-apoptotic cell death mechanism of lapatinib in A549 cells. This is evidenced by the following observations: i) lapatinib alone triggered cell death, but did not affect $\Delta \psi_{\mathrm{m}}$; ii) lapatinib-mediated cell death could not be prevented by z-VAD-fmk at even higher concentration; iii) lapatinib produced a loss of membrane integrity without nuclear fragmentation; iv) lapatinib induced mitochondrial swelling. Strikingly, however, cotreatment with
HDACi sensitized cells for apoptosis induction by lapatinib: While lapatinib alone did not affect $\Delta \psi_{\mathrm{m}}$, in combination with HDACi it induced a concentration-dependent loss of $\Delta \psi_{\mathrm{m}}$. Moreover, z-VAD-fmk protected cells from HDACi/ lapatinib-induced $\Delta \psi_{\mathrm{m}}$ dissipation, whereas it had no effect on cell death.

Taken together, these observations point to the following scenario: A549 cells are initially unresponsive to apoptosis 
induction by all three TKI applied, erlotinib, gefitinib and lapatinib, but responsive to non-apoptotic cell death induction by the latter. Resistance to apoptosis induction can in all cases be overcome by cotreatment with HDACi; in case of lapatinib however, apoptosis induction is masked by non-apoptotic cell death, as judged by PI uptake analysis. Support for the general usefulness of HDACi in overcoming drug resistance comes from a recent work, in which it has been shown that co-exposure to HDACi prevented the development of resistance not only against erlotinib, but also against the RAF kinase inhibitor AZ628 and the cytostatic cisplatin (27).

In conclusion, we have shown that erlotinib- and gefitinibresistant KRAS-mutated NSCLC cells can be killed by either combination treatment with HDACi or single treatment with lapatinib. Hence, both treatment regimens are well worth considering for the therapy of NSCLC bearing KRAS mutations.

\section{Acknowledgements}

We thank Dr Annett Eitner (Department of Anatomy II, University Hospital Jena) for her help with confocal laser scanning microscopy. We also thank Sabine Becker and Susann Wittig for their excellent technical assistance. This work was supported by the 'Wilhelm Sander-Stiftung, Neustadt/Donau'.

\section{References}

1. Herbst RS, Heymach JV and Lippman SM: Lung cancer. N Engl J Med 359: 1367-1380, 2008.

2. Ciardiello $\mathrm{F}$ and Tortora G: EGFR antagonists in cancer treatment. N Engl J Med 358: 1160-1174, 2008.

3. Mok TS, Wu YL, Thongprasert S, et al: Gefitinib or carboplatinpaclitaxel in pulmonary adenocarcinoma. N Engl J Med 361: 947-957, 2009

4. Linardou H, Dahabreh IJ, Kanaloupiti D, Siannis F, Bafaloukos D, Kosmidis P, Papadimitriou CA and Murray S: Assessment of somatic k-RAS mutations as a mechanism associated with resistance to EGFR-targeted agents: a systematic review and meta-analysis of studies in advanced non-small-cell lung cancer and metastatic colorectal cancer. Lancet Oncol 9: 962-972, 2008.

5. Moy B, Kirkpatrick P, Kar S and Goss P: Lapatinib. Nat Rev Drug Discov 6: 431-432, 2007.

6. Lane AA and Chabner BA: Histone deacetylase inhibitors in cancer therapy. J Clin Oncol 27: 5459-5468, 2009.

7. Richon VM, Garcia-Vargas J and Hardwick JS: Development of vorinostat: current applications and future perspectives for cancer therapy. Cancer Lett 280: 201-210, 2009.

8. Nolan L, Johnson PW, Ganesan A, Packham G and Crabb SJ: Will histone deacetylase inhibitors require combination with other agents to fulfil their therapeutic potential? Br J Cancer 99 689-694, 2008.

9. Sonnemann J, Huls I, Sigler M, Palani CD, Hong IT, Volker U, Kroemer HK and Beck JF: Histone deacetylase inhibitors and aspirin interact synergistically to induce cell death in ovarian cancer cells. Oncol Rep 20: 219-224, 2008.

10. Dzieran J, Beck JF and Sonnemann J: Differential responsiveness of human hepatoma cells versus normal hepatocytes to TRAIL in combination with either histone deacetylase inhibitors or conventional cytostatics. Cancer Sci 99: 1685-1692, 2008.
11. Palani CD, Beck JF and Sonnemann J: Histone deacetylase inhibitors enhance the anticancer activity of nutlin-3 and induce p53 hyperacetylation and downregulation of MDM2 and MDM4 gene expression. Invest New Drugs 2010. Epub ahead of print; DOI: $10.1007 / \mathrm{s} 10637-010-9510-7$.

12. Edwards A, Li J, Atadja P, Bhalla K and Haura EB: Effect of the histone deacetylase inhibitor LBH589 against epidermal growth factor receptor-dependent human lung cancer cells. Mol Cancer Ther 6: 2515-2524, 2007.

13. Zhang W, Peyton M, Xie Y, Soh J, Minna JD, Gazdar AF and Frenkel EP: Histone deacetylase inhibitor romidepsin enhances anti-tumor effect of erlotinib in non-small cell lung cancer (NSCLC) cell lines. J Thorac Oncol 4: 161-166, 2009.

14. Witta SE, Gemmill RM, Hirsch FR, et al: Restoring E-cadherin expression increases sensitivity to epidermal growth factor receptor inhibitors in lung cancer cell lines. Cancer Res 66: 944-950, 2006.

15. Witta SE, Dziadziuszko R, Yoshida K, Hedman K, VarellaGarcia M, Bunn PA Jr and Hirsch FR: ErbB-3 expression is associated with E-cadherin and their coexpression restores response to gefitinib in non-small-cell lung cancer (NSCLC). Ann Oncol 20: 689-695, 2009.

16. Rusnak DW, Alligood KJ, Mullin RJ, et al: Assessment of epidermal growth factor receptor (EGFR, ErbB1) and HER2 (ErbB2) protein expression levels and response to lapatinib (Tykerb, GW572016) in an expanded panel of human normal and tumour cell lines. Cell Prolif 40: 580-594, 2007.

17. Diaz R, Nguewa PA, Parrondo R, et al: Antitumor and antiangiogenic effect of the dual EGFR and HER-2 tyrosine kinase inhibitor lapatinib in a lung cancer model. BMC Cancer 10: 188, 2010.

18. Chou TC: Drug combination studies and their synergy quantification using the Chou-Talalay method. Cancer Res 70: 440-446, 2010.

19. Valenzuela DM and Groffen J: Four human carcinoma cell lines with novel mutations in position 12 of c-K-ras oncogene. Nucleic Acids Res 14: 843-852, 1986.

20. Ono M, Hirata A, Kometani T, Miyagawa M, Ueda S, Kinoshita H, Fujii T and Kuwano M: Sensitivity to gefitinib (Iressa, ZD1839) in non-small cell lung cancer cell lines correlates with dependence on the epidermal growth factor (EGF) receptor/extracellular signal-regulated kinase $1 / 2$ and EGF receptor/Akt pathway for proliferation. Mol Cancer Ther 3: 465-472, 2004.

21. Janmaat ML, Rodriguez JA, Gallegos-Ruiz M, Kruyt FA and Giaccone G: Enhanced cytotoxicity induced by gefitinib and specific inhibitors of the Ras or phosphatidyl inositol-3 kinase pathways in non-small cell lung cancer cells. Int J Cancer 118: 209-214, 2006.

22. Shimizu S, Eguchi Y, Kamiike W, et al: Induction of apoptosis as well as necrosis by hypoxia and predominant prevention of apoptosis by Bcl-2 and Bcl-XL. Cancer Res 56: 2161-2166, 1996.

23. Johnson LV, Walsh ML, Bockus BJ and Chen LB: Monitoring of relative mitochondrial membrane potential in living cells by fluorescence microscopy. J Cell Biol 88: 526-535, 1981.

24. Galluzzi L, Aaronson SA, Abrams J, et al: Guidelines for the use and interpretation of assays for monitoring cell death in higher eukaryotes. Cell Death Differ 16: 1093-1107 2009.

25. Gong Y, Somwar R, Politi K, Balak M, Chmielecki J, Jiang X and Pao W: Induction of BIM is essential for apoptosis triggered by EGFR kinase inhibitors in mutant EGFR-dependent lung adenocarcinomas. PLoS Med 4: e294, 2007.

26. Kouraklis G and Theocharis S: Histone deacetylase inhibitors: a novel target of anticancer therapy (Review). Oncol Rep 15: 489-494, 2006

27. Sharma SV, Lee DY, Li B, Quinlan MP, et al: A chromatin-mediated reversible drug-tolerant state in cancer cell subpopulations. Cell 141: 69-80, 2010. 